\title{
A conditioned weight illusion: Reafference learning without a correlation store
}

\author{
WAYNE HERSHBERGER and GIOVANNI MISCEO \\ Northern Illinois University, DeKalb, Illinois
}

\begin{abstract}
Each of 20 female college students repeatedly judged the weight of a heavy metal cylinder dropped repeatedly (180 times) into her waiting hand. The cylinder came to appear lighter to her when the release of the cylinder was accompanied by the onset of an indicator lamp than when it was not, but only providing the onset of the lamp preceded the release of the cylinder by a half second rather than being simultaneous with it. This conditional illusion has implications for von Holst and Mittelstaedt's well-known thesis that every neural efference leaves an efference copy or corollary discharge of itself in the nervous system to be compared with concurrent neural reafference. Apparently some types of conditioned efference leave no copies, and objects lifted in part by such unregistered efforts appear lightened accordingly. The illusion illustrates a form of reafference learning that need involve no correlation store (Held, 1961).
\end{abstract}

This paper reports a simple experimental demonstration of a classically conditioned illusory sense of effort required to suspend a hefted object, a phenomenon that has interesting theoretical implications for notions of sensed efference. Such terms as sensed efference, "effort of will" (Helmholtz, 1866/1962), "corollary discharge"' (Sperry, 1950), "efference copy" (von Holst \& Mittelstaedt, 1950), or the like, refer generally to the notion that palpable sensory impressions may result from the monitoring of efferent motor impulses as well as from afferent sensory impulses. The notion is not new. It has long been controversial. Helmholtz and James argued for and against it, respectively (Festinger \& Canon, 1965), and the controversy persists to this day (Goodwin, McCloskey, \& Matthews, 1972; MacKay \& Mittelstaedt, 1974; Matin, 1982; Shebilski, 1977a). However, the possibility remains that disputants on all sides are right, that some types of efference are sensed whereas others are not. Indeed, even before the turn of the century, G. E. Muller was explaining Charpentier's (1891) classic size-weight illusion in just such terms (Martin \& Muller, 1899). It was our purpose to test Muller's ideas and explore their implications for what is perhaps the best known contemporary theory of sensed efference: von Holst and Mittelstaedt's efference-copy hypothesis (1950).

Von Holst and Mittelstaedt were concerned with the fact that an organism's detection of environ-

This work was presented in part at the 54th Annual Convention of the Midwestern Psychological Association, Minneapolis, Minnesota, May 6-8, 1982. Reprint requests should be submitted to Wayne Hershberger, Department of Psychology, Northern Illinois University, DeKalb, Illinois 60115. Giovanni Misceo is now at the Department of Psychology, Kansas State University, Manhattan, Kansas 66502. mental disturbances (such as the weight of an object dropped into the hand) is generally complicated by the fact that the organism's own actions impact the very exteroceptor(s) by which the environmental disturbance is to be detected. For example, the position or motion of a load-bearing arm is a joint function of external gravitational forces and internal muscular forces. They termed this complication the reafference principle, and the afference resulting from external and internal sources (e.g., gravitational and muscular forces) ex- and reafference, respectively. The reafference principle implies that the veridical detection of any environmental disturbance, entailing, as it does, the achievement of pure exafference, presupposes an accurate neural accounting of and compensation for contaminating reafference. It was their hypothesis that this compensation for reafference is realized by means of a putative efference copy, a corollary discharge of neural efference, which hypothetically (1) is an exact copy of concurrent reafference, and (2) when subtracted neurally from afference yields pure exafference as a remainder. This remainder, being pure exafference, serves to mediate veridical percepts of environmental disturbances. Although this solution to the perceptual predicament is parsimonious, a problem persists: The presumed equivalence between an efference copy and its conjugate reafference is violated by the fact that the reafferent consequences of a given efference may change from time to time as a function of circumstance, say from muscular fatigue or the like. If von Holst and Mittelstaedt's functional schema is to mediate veridical percepts of environmental disturbances, it must act automatically to resolve any such discordance. Two possibilities exist: (1) either the efference copies may be altered automatically to correspond to their conjugate reafferences, or (2) the reafferences may be altered automatically to correspond to their conjugate efference copies. 
Twenty years ago, Richard Held (1961) hypothesized the former alternative. Held postulated a neural correlation storage mechanism that allowed the central nervous system to monitor the efference/ reafference transfer function of its environment and utilize this empirically determined function (or "correlation") to generate corresponding efference copies." Held's postulate spawned a host of ingenious perceptual (Welch, 1978) and developmental (Rosinski, 1977) studies, which, on the whole, serve convincingly to demonstrate that organisms do adapt to the idiosyncrasies of their particular environments. However, these diverse demonstrations do not necessarily warrant the theory that spawned them, for there is the alternate possibility (2), noted above, which we advance here as a working hypothesis. Our hypothesis requires not Held's putative correlation store, but Müller's two types of efference: (1) a type of efference that is registered as efference copies, and (2) another, unregistered type of efference that contributes only to reafference, leaving nothing to efference copies. (For example, Matin, 1972, 1976, 1982 , has advanced a "hybrid theory" of the oculomotor system in which only gamma efference contributes to a copy of registered eye position, with alpha motor neurons contributing only to reafference.) Our hypothesis requires also that (1) the unregistered efference be docile, that is, amenable to conditioning, and that (2) the conditioning be automatic or reflexive. In effect, our hypothesis implies the possibility of classically conditioning a type of unregistered efference akin to that which is responsible, according to Muiller, for the size-weight illusion.

Charpentier's size-weight illusion (Granit, 1972) refers to the fact that for any lifted object of constant weight, the object's heaviness decreases approximately as a logarithmic function of the object's volume (Stevens \& Rubin, 1970). Müller, who believed that the perceived weight of an object depends upon the relative ease with which it can be lifted (Müller \& Schuman, 1889), characterized the size-weight illusion as an illusory sense of effort (Martin \& Müller, 1899). Müller's thesis comprises, essentially, three hypotheses: (1) There is a sense of effort corresponding to intended, rather than actual, muscular exertion. (2) One learns from experience to exert more force in lifting larger objects. And, (3) at least some of the force one learns to exert is unintended, that is, is exerted unwittingly as a form of unsensed effort. Taken together, these three hypotheses account for the size-weight illusion as follows: If the larger of two objects of equal weight is lifted with more force, as one has learned, at least in part, to do unwittingly (i.e., with no sense of effort), then it follows that the larger of the two equally heavy objects will be lifted with greater ac- celeration and terminal velocity and, hence, appear lighter.

Recent research with lifted weights has provided data consistent with one or more of each of these three hypotheses. As for the first hypothesis, McCloskey, Ebeling, and Goodwin (1974) have concluded, from several experiments, that there is a centrifugal "sense of effort" that is separate from a peripheral sense of muscular tension. In one experiment, they asked subjects pulling upon an unyielding lever either to exert a constant effort of contraction or to maintain a constant level of tension while they, the experimenters, reflexively altered that tension by stimulating the tendon of the agonist or antagonist with a vibrator. (Vibrating a muscle stimulates its primary spindle afferents, reflexively exciting the vibrated muscle and inhibiting its antagonist.) They found that their subjects could alternatively (1) compensate for the involuntary innervation, keeping tension constant, or (2) maintain a constant level of effort, allowing the reflexive innervation to temporarily alter tension but only to have it return to its original value upon removal of the vibrator. They concluded that their subjects were "able to modify either [tension or sensed effort] so as to keep the other constant."

Davis and his associates have reported data that are consistent with Muller's second hypothesis. They found that when the larger of two lifted objects of equal weight is judged to be the lighter, (1) the muscular action potential recorded $250 \mathrm{msec}$ prior to each lift is greater for the larger object (Davis \& Brickett, 1977), and (2) the acceleration and peak velocity of the object during each lift is greater for the larger object (Davis \& Roberts, 1976).

Finally, the third hypothesis is plausible in view of the aforementioned finding (McCloskey et al., 1974) that the reflexive isometric contractions produced by stimulating a muscle with a vibrator yields an impression of increased tension rather than an impression of increased effort. Or the vibration "produces an illusion of movement [of the affected joint] ... in the direction which would occur if the vibrated muscle was being stretched" (Goodwin et al., 1972). For example, "when the wrist flexor muscle is vibrated and the wrist is passively extended to a position close to its anatomical limit, the hand is felt to be bent [backward toward the dorsal surface of the forearm] to a position about $29 \mathrm{deg}$ beyond its maximum operating range"' (Craske, 1977).

If reflexive contractions elicited by an unconditional stimulus (vibration) do not register as realized efference, then, presumably, reflexive contractions elicited by conditional stimuli would not do so either. A word of caution: We are asserting here not that there are no conscious sensations arising from the activity of muscle spindles, but rather that such sen- 
sations register afference, not efference. Since the afference from an intrafusal fiber immediately becomes the efference for the spindle's extrafusal counterpart, it is not immediately clear whether a conscious sensation arising from the activity of a muscle spindle in a gamma motor loop is to be regarded as sensed afference or sensed efference. However, judging from the nature of the kinesthetic illusion resulting from vibration of an isometric muscle, the former alternative (sensed afference) appears to be the more reasonable one. That is, the kinesthetic illusion resulting from the vibration of an isometric muscle is the impression that the muscle is lengthening, not shortening, directly reflecting the stretching of the muscle spindle receptor rather than the imminent contraction of the extrafusal muscle fibers (Craske, 1977; Goodwin et al., 1972; Granit, 1972).

Of course, muscle-spindle output tends only to provide an index of the degree to which extrafusal muscular contractions fall short of the intrafusal standards commanded by gamma efferents; hence, spindle output might well be regarded as a copy of unrealized efference, but a copy of efference nonetheless. (The fact that such a copy of unrealized efference proceeds centripetally along afferent neurons does not mitigate the fact that it is a copy of unrealized efference, or an efference copy, that is being transmitted. ${ }^{2}$ ) However, whether muscle-spindle output is regarded simply as afference or as a copy of unrealized efference, it clearly does not register centrally as the realization of the reflexive muscular contractions it, itself, serves to elicit. Those elicited reflexes appear to go unregistered as such and, hence, constitute a form of unsensed efference.

In concert, the aforementioned research findings, each fully consonant with Muller's thesis, imply the possibility of classically conditioning illusory impressions akin to those characterizing Charpentier's size-weight illusion. The present experiment realized this possibility by demonstrating that a heavy metal cylinder dropped into the hand of a waiting subject comes to appear lighter on those occasions when the release of the cylinder is accompanied by the onset of an indicator lamp than when it is not, but only providing the onset of the lamp precedes the release of the cylinder by $.5 \mathrm{sec}$ rather than being simultaneous with it.

\section{METHOD}

\section{Subjects}

Twenty females enrolled in an introductory psychology course at Northern Illinois University volunteered to serve as subjects and earned bonus credit for participating in the experiment.

\section{Procedure}

Each subject worked alone in a seated position and supported the weight to be judged in the palm of her preferred hand while keeping her forearm roughly parallel to the floor and her upper arm roughly parallel with her torso. Each subject was led to believe she was judging the relative weights of two very similar objects while, in fact, she was judging the same object on each trial. The object was a metal cylinder with a diameter of $7.3 \mathrm{~cm}$, a length of $6.4 \mathrm{~cm}$, and a weight of $1.9 \mathrm{~kg}$. To begin each trial, 180 in all, the experimenter suspended the cylinder from an electromagnet situated in the subject's median sagittal plane about $25 \mathrm{~cm}$ in front of her torso and about $15 \mathrm{~cm}$ above her knees. During each trial, the cylinder was abruptly released into the palm of the subject's hand, held immediately beneath it, and she was required to say, or guess, whether it was the lighter or heavier of the "two" cylinders she was expected to discriminate. The abrupt release of the cylinder into her hand served as an unconditional stimulus, UCS, for unspecified reflexive muscular contractions. While awaiting the UCS, the subject visually fixated a small lamp which was periodically illuminated to provide a conditional stimulus, CS, for reflexive muscular contractions. The lamp, situated at eye level directly in front of the subject at a distance of about $50 \mathrm{~cm}$, was a 24-V dc instrument-panel indicator lamp with a green lens about $2 \mathrm{~cm}$ in diameter. Illumination of the lamp for a .5-sec period served as the CS.

Either a forward or a simultaneous conditioning paradigm was used for a given subject. In the former case, the interstimulus interval, ISI, was $.5 \mathrm{sec}$; that is, the lamp came on $.5 \mathrm{sec}$ before the UCS and went off simultaneously with UCS onset. In the latter case, CS and UCS onsets were simultaneous and the lamp went off $.5 \mathrm{sec}$ later. A system of logic modules (Lehigh Valley Electronics) was used to time the conditioning intervals and automatically administer the conditioning stimuli. The UCS was presented by interrupting an electronic circuit $(24 \mathrm{~V} \mathrm{dc})$ through the electromagnet for a period of $40 \mathrm{msec}$.

One half of the subjects, the experimental group, were conditioned using the forward paradigm; the other half, the control group, were conditioned using the simultaneous paradigm, known to be remarkably ineffective (Hall, 1976). Each subject received 180 trials total, with the UCS presented on every trial. The CS was presented only on every other trial: either even-numbered or odd-numbered trials, as determined randomly for each subject. The intertrial intervals were $20 \mathrm{sec}$ to begin with and were reduced as the subject became accustomed to the task; more specifically, they were $20 \mathrm{sec}$ for the first five trials, $15 \mathrm{sec}$ for the next five, $12 \mathrm{sec}$ for the next five, and $10 \mathrm{sec}$ for the remaining trials.

\section{Instructions}

The experimenter began each experimental session by handing the subject two similar metal cylinders and telling her that, although they might seem identical, one was a bit lighter than the other and that her task would be to detect the difference by judging the weights one at a time and to respond either "lighter" or "heavier." She was told she would have to guess the first few times. Then the experimenter placed both cylinders behind a screen in order to hide his selection of the experimental cylinder, which he suspended from the electromagnet for the first trial. After the first and all subsequent trials, the experimenter replaced the cylinder behind the screen in order to hide the fact that he selected the same cylinder for each trial. The subject was instructed to respond promptly on each trial-to report first impressions-and to immediately hand the cylinder to the experimenter rather than hold and heft it. Then, as soon as the experimenter had again suspended the cylinder from the electromagnet, he asked the subject to cup it lightly in her palm as she awaited the next trial. She was asked to watch the indicator lamp so that, ostensibly, it could be determined whether such a stimulus helped or hindered her performance.

\section{RESULTS}

The experimental task was scored as a signaldetection task, with the conditional reflex, $\mathbf{C R}$, or 
rather the putative illusory lightness it occasions, being the signal detected. Assuming that the strength of the CR might vary from trial to trial, we scored the data as though a CR, however negligible, always occurred on CS trials and never occurred on nonCS trials. That is, a "lighter" judgment on a CS trial was scored as a hit and a "lighter" judgment on a non-CS trial was scored as a false alarm. Now, if the signal being detected is an illusory CR, the strength of this signal, computed as $\mathrm{d}^{\prime}$, should increase as conditioning proceeds, but only for the experimental group.

The judgments of each subject were partitioned into six consecutive blocks of 30 trials each, and values of $d^{\prime}$ and $B$ (subjects' response criteria) were computed for each block. In each case, a frequency of one was first added to each cell of the fourfold matrix of trial outcomes (hit, miss, false alarm, and correct rejection); otherwise, an occasionally empty cell would have prevented our computing $d^{\prime}$ and $B$ for particular blocks of trials.

\section{Analysis of d'}

For the experimental group (.5-sec ISI), the average $d$ 's for Blocks $1-6$ were $.33, .80,1.48,1.25$, 1.67 , and 1.67, respectively. For the control group, the average $d^{\prime} s$ for the six blocks were, respectively, $-.30, .05,-.32,-.31,-.23$, and -.55 .

A two-way analysis of variance of the data summarized by these 12 means yielded three significant effects: a main effect of interstimulus interval $[F(1,18)$ $=26.63, \mathrm{p}<.01]$, a main effect of training block $[F(5,90)=5.05, p<.01]$, and an interstimulus interval $\times$ training block interaction $[F(5,90)=9.06, p<$ $.01]$. The interaction had only one significant component: For the .5 -sec ISI, the simple main effect of training block was significant $[F(5,90)=12.48$, $\mathrm{p}<.001$ ]; for the simultaneous condition, the simple main effect of training block was not significant $[F(5,90)=1.57, p>.1]$. Neither does the overall mean $\mathrm{d}^{\prime}$ for the simultaneous condition $(-.28)$ differ significantly from zero $[t(9)=-.56, p>.5]$.

\section{Analysis of B}

A two-way analysis of variance of the subject's response criteria, B, yielded no significant effects ( $p>$ $.05)$. The value of $B$ employed by the average subject, overall, was .9, not far from the value of 1.0 which might be expected of an ideal observer given our instructions.

\section{DISCUSSION}

The results are fully consistent with Muller's notion that one may learn to exert effort unwittingly and experience weight illusions as a result. The performance of our experimental group, tested under conditions conducive to classical conditioning, reflects an acquired weight illusion, whereas the performance of our control group, subjected to a conditioning paradigm known to be remarkably, if not altogether, ineffective (Hall, 1976), does not. The illusion is an apparent difference in the weight of the cylinder on the signaled and unsignaled trials. In deference to Muller, we have called this difference an illusory lightness (of the cylinder on the signaled trials), but we could just as well have called it an illusory heaviness (on the unsignaled trials). What our data demonstrate is not that unsensed efference in the form of a conditioned reflex improves or impairs performance but only that it alters perception in a predictable manner, as suggested by Muller. We will consider presently how such alterations may affect perceptual adaptation, but first let us reconsider our principal theoretical issue.

If von Holst and Mittelstaedt's functional schema is to mediate veridical percepts of environmental disturbances, it must act to resolve automatically any circumstantial discordance between efference copies and their conjugate reafferences, either (1) by altering its efference copies to correspond to their conjugate reafferences, or (2) by altering its reafferences to correspond to their conjugate efference copies. Held advanced the former alternative in the form of a putative correlation storage mechanism. We are advancing the latter alternative in terms of Müller's notion of unsensed effort. According to our thesis, the relationship between the efference copy and its conjugate reafference is simply not allowed to vary, thereby rendering any correlation storage mechanism superfluous. The process requires merely a type of efference which contributes only to reafference, leaving nothing to efference copies, viz, unregistered conditional and unconditional reflexes. In other words, by adding a type of unregistered efference (either excitatory or inhibitory) to the efference that is registered (does yield efference copies), classical conditioning would tend to maintain an equivalence between efference copies and their conjugate reafferences, providing only that any momentary lack of correspondence serve as an unconditional stimulus for reflexive compensation, a provision von Holst and Mittelstaedt not only incorporated into their original functional schema, but also sought to illustrate with several experimental demonstrations: for example, the interminable optokinesis of a fly whose head had been rotated $180 \mathrm{deg}$. If they go unregistered as efference copies, these unconditional compensatory reflexes, together with the conditional compensatory reflexes they would inevitably reinforce, should serve to maintain, by the principle of negative feedback, an efference-copy/reafference equivalence, all without benefit of Held's putative storage mechanism. 
Held's hypothesis appears to be an essential theoretical addition to that of von Holst and Mittelstaedt only when applying their functional schema to descriptions of such uncommon motoneuronal mechanisms as the electric organ discharge of the mormyrid family of electric fish. The electric organ discharge is an unusual efference in that the brief volley from motoneurons to the electric organ is always the same. That is, it is never modulated by compensatory reflexes, either registered or unregistered. Hence, the resolution of any momentary discordance between an efference copy and its conjugate reafference would require a change in the efference copy, not the reafference. Bell (1981) has recently reported evidence of just such an "adaptive efference copy" in the ampullary electroreceptors in the mormyrid species Gnathonemus petersii. Bell's data appear to be conclusive evidence for Held's hypothesis, but it is severely limited in its scope. As Bell himself aptly notes, "ordinary motor systems ... where the output is graded and complex" are an altogether different matter. His caveat appears particularly cogent in view of the present demonstration that reflexive components of an ordinary efference complex may go unregistered, contributing to reafference but not to efference copies.

The possibility remains, for simple and complex neuromotor systems alike, that some sort of neural correlator does perhaps monitor and coordinate paired reafferences such as visually fixating one's visible hand (e.g., Hardt, Held, \& Steinbach, 1971). Although Held's (1961) hypothesis concerns efference/reafference coordination, his eye/hand (eye/ paw) coordination experiments have concerned what might perhaps best be called efference/efference coordination, or reafference/reafference coordination. In other words, much of Held's research appears unrelated to his theory (cf. Welch, 1978, p. 28). Conversely, much the same can be said of the research reported in its refutation. For reviews of the controversy, see: Harris (1965), Kornheiser (1976), and Welch (1978). We agree with Hay, for instance, that eye/hand coordination differs significantly from visual position constancy, VPC, the phenomenon that provides perhaps the "best exemplification of reafference learning" (Hay, 1981). Hay's VPC research employs a method similar to that conceived by Wallach and Kravitz (1965). A luminous spot in a dark room is made to undergo horizontal oscillations when Hay's subject nods his head up and down. Eventually this oscillating spot will come to appear stationary to the subject and a luminous spot which is actually stationary will appear to move horizontally as he nods his head. Hay (1968), noting that this type of adaptation depends on the subject's following the luminous spot with his eyes, proposed that the adaptation involves the learning of "compensatory eye movements." He seems thus to have anticipated us in arguing that "reafference learning," as he calls it, may involve the acquisition or alteration, not of expectations (or efference copies), but of unregistered efference which helps realize such expectations (cf. Rock, 1966, p. 117). However, Hay continues, paradoxically, to advance his data as evidence for Held's hypothesis. Hay (1981) writes, "In reafference learning, a person learns to expect a certain stimulus movement (Held, 1961; Welch, 1978)." This statement is consistent with Held's hypothesis but inconsistent with Hay's data (cf. Rock, 1966, p. 117). At the end of reafference training, Hay's subject not only expects, but sees, no stimulus change as he nods his head, even though the spot is made to oscillate at every nod. (To argue that compensatory eye movements imply expectations of stimulus changes is to beg the question.)

Comparable findings have been reported by McLaughlin, Kelly, Anderson, and Wenz (1968) and Pola (1976), using McLaughlin's (1967) technique for conditioning parametric adjustments of saccadic eye movements. McLaughlin (1967) found it possible to condition a reduction in the size of saccades used to shift fixation from target $A$ to target $B$ by extinguishing $B$ during each saccade and replacing it with a surrogate target $\mathbf{B}^{\prime}$ situated closer to $A$. McLaughlin et al. (1968) found that, with targets B and $B^{\prime}$ situated 10 and 5 deg from $A$, respectively, initial saccadic amplitude diminished from a mean value of 9.43 to $5.80 \mathrm{deg}$ after only 11 conditioning trials. However, the registered or perceived size of the saccade did not appear to change, for, when these conditioned subjects subsequently maintained fixation of target $A$ and pointed toward the apparent location of the eccentric target B, neither B nor B' being visible at the time, they pointed in the same direction they had before conditioning. Thus, the parametric adjustment of the saccadic eye movements appeared to involve a form of unsensed efference, with sensed efference remaining unchanged. ${ }^{3}$ Miller (1980) has argued that Pola's (1976) findings may be similarly understood. Using McLaughlin's conditioning technique and Matin's (1972) psychophysical procedures for determining the retinal PSE of target $A$ during saccades from $\mathrm{A}$ to $\mathrm{B} / \mathrm{B}^{\prime}$, Pola found that, whereas the size of a normal 8-deg saccade could be conditionally reduced to, say, $5 \mathrm{deg}$, the accompanying shift of target A's retinal PSE still resembled that which normally accompanies an 8-deg saccade. Assuming, as we do, that the saccade's reduction from 8 to 5 deg reflects a form of unsensed inhibitory efference, it follows, as Miller has noted, that "the central efferent command to fixate the $8^{\circ}$ peripheral target may be unchanged from that prior to preconditioning, so that the perceptual system would assume that an $8^{\circ}$ saccade had been made on the 
basis of extraretinal information" (Miller, 1980). This notion that the compensatory efference involved in oculomotor conditioning is a form of unsensed efference is documented even further by Miller and Festinger's (1977) compelling demonstration that whatever perceptual adaptation as does occur to the optical curvature produced by viewing the environment through wedge prisms does not likely stem from any perceptual registration of the subject's altered eye movements under these circumstances, but rather is probably but an instance of Gibson's (1933) normalization effect. And, although there is evidence to the contrary (Held \& Rekosh, 1963), that evidence does not appear replicable (Gyr, Willey, \& Henry, 1979).

Now, although the notion of unsensed conditional efference does not imply the possibility of perceptual adaptation to any and all types of abnormal reafference (optical curvature, for instance), it surely implies the possibility of some types, for the notion is but the complement of Harris's (1965) hypothesis. It was Harris's notion that perceptual adaptation involves an altered sense of position, whereas we are advancing the notion of an unsensed altered position. And, although Harris's notion is inconsistent with the data reviewed immediately above, those forms of perceptual adaptation that are consistent with Harris's hypothesis should by and large be consistent with ours.

Hay's experiments described above illustrate nicely the perceptual adaptation effected by unsensed conditioned efference: Normally, when one simply nods, stationary distal objects in the median sagittal plane change their elevation relative to the head but not their azimuth relative to the eye. By making azimuth changes contingent upon head nodding, Hay has added to the normal reafference (elevation changes) an abnormal reafference (azimuth changes). The subject's initial perception of these azimuth changes as environmental disturbances (motions) is illusory, since, for the subject, they are reafferent rather than exafferent changes. (This point would perhaps be more obvious, but certainly no more valid, had Hay left the luminous spot stationary and arranged to have head nodding shift the azimuth merely of its virtual image by means of reflection or refraction.) With practice, Hay's subjects developed compensatory eye movements whose normal reafference opposed the abnormal, head-nodding reafference he had arranged. To the degree that these two types of reafference (abnormal and compensatory) are equal and opposite, they serve to cancel each other, leaving the original normal reafference (elevation changes) intact. Furthermore, if the neural efference which produces the conditioned compensatory eye movements goes unregistered, as appears to be the case, the efference copy of the head nodding would remain true to its restored conjugate reafference so that they would cancel each other, leaving no residual. Hence, the subject should come to perceive the luminous spot as stationary when he nods his head, just as Hay found. This perception of no environmental disturbance (motion) is veridical for the subject, since all motions of the luminous spot are self-produced. (One person's reafference is another person's exafference; it would be illusory for a bystander to see the luminous spot as stationary, but that is beside the point. $)^{4}$

Hay is not the only investigator to show that unregistered oculomotor efference may be involved in perceptual adaptation. Ebenholtz and his colleagues (Ebenholtz, 1970, 1974, 1981; Ebenholtz \& Fisher, 1982; Ebenholtz \& Wolfson, 1975; Paap \& Ebenholtz, 1976, 1977; Shebilski, 1977b) have implicated muscle potentiation as a contributing factor in several forms of perceptual adaptation, including adaptation to prismatic displacement. Consider, for example, the case of a subject wearing base-left prisms which shift the visual scene to the right. The subject may be expected to maintain his gaze predominantly to the right, thereby potentiating the lateral rectus of the right eye and the medial rectus of the left eye. ["Muscle potentiation refers to an involuntary unregistered component of continued innervation in the direction of previous muscular stimulation" (Paap \& Ebenholtz, 1976).] Hence, subsequently, when the subject attempts to look straight ahead, his gaze will involuntarily be shifted to the right, automatically compensating for the rightward shift of the visual scene produced by the base-left prisms. The potentiation thus proves to be adaptive. Although the potentiation is conditional upon the circumstances that induce it and is, hence, a form of unsensed conditional efference, the conditioning depends upon exercise, rather than practice, and dissipates merely with rest, but not so rapidly as to preclude negative aftereffects, the hallmark of perceptual adaptation. When the prisms are first removed, the abnormal retinal reafference resulting from the potentiated rotation of the eyes persists and is perceived as an illusory rightward displacement of the environment.

The various findings of Ebenholtz and his colleagues, as well as those of Hay, appear to parallel perfectly our hypothesized mechanism resolving circumstantial discordance between efference copies and their conjugate reafferences. That is, the very circumstances which generate such discord serve also to condition its resolution in the form of involuntary, unregistered, compensatory efference. With rare exceptions (see Bell above), a correlation store such as Held hypothesized appears unnecessary. 


\section{REFERENCES}

BeLl, C. C. An efference copy which is modified by reafferent input. Science, 1981, 214, 450-453.

Charpentier, A. Analyses experimentale de quelques elements de la sensation de poids. Archieves de Physiologie Normal et Pathologique, 1891, 3, 122-135.

Craske, B. Perception of impossible limb positions induced by tendon vibration. Science, 1977, 196, 71-73.

Davis, C. M., \& BricketT, P. The role of preparatory muscular tension in the size-weight illusion. Perception \& Psychophysics, 1977, 22, 262-264.

Davis, C. M., \& Roberts, W. Lifting movements in the sizeweight illusion. Perception \& Psychophysics, 1976, 20, 33-36.

EвenholTz, S. M. On the relation between interocular transfer of adaptation and Hering's law of equal innervation. Psychological Review, 1970, 77, 343-347.

Ebenholtz, S. M. The possible role of eye-muscle potentiation in several forms of prism adaptation. Perception, 1974, 3, 477-485.

Ebenholtz, S. M. Hysteresis effects in the vergence control system: Perceptual implications. In D. F. Fisher, R. A. Monty, \& J. W. Senders (Eds.), Eye movements: Visual perception and cognition. Hillsdale, N.J: Erlbaum, 1981.

Ebenholtz, S. M., \& Fisher, S. K. Distance adaptation depends upon plasticity in the oculomotor control system. Perception \& Psychophysics, 1982, 31, 551-560.

Ebenholtz, S. M., \& Wolfson, D. M. Perceptual aftereffects of sustained convergence. Perception \& Psychophysics, 1975, $17,485-491$.

Festinger, L., \& Canon, L. K. Information about spatial location based on knowledge about efference. Psychological Review, 1965, 72, 373-384.

GiBson, J. J. Adaptation, after-effect and contrast in perception of curved lines. Journal of Experimental Psychology, 1933, 16, $1-31$.

Goodwin, G. M., McCloskey, D. I., \& Matthews, P. B. C. The contribution of muscle afferents to kinesthesia shown by vibration induced illusions of movement and by the effects of paralysing joint afferents. Brain, 1972, 95, 705-748.

Granit, R. Constant errors in the execution and appreciation of movement. Brain, 1972, 95, 649-660.

Gyr, J., Willey, R., \& Henry, A. Motor-sensory feedback and geometry of visual space: An attempted replication. The Behavioral and Brain Sciences, 1979, 2, 59-94.

HALl, J. F. Classical conditioning and instrumental learning. New York: Lippincott, 1976.

Hallett, P. E., \& Lightstone, A. D. Saccadic eye movements towards stimuli triggered by prior saccades. Vision Research, 1976, 16, 99-106. (a)

Hallett, P. E., \& Lightstone, A. D. Saccadic eye movements to flashed targets. Vision Research, 1976, 16, 107-114. (b)

Hardt, M. E., Held, R., \& Steinbach, M. J. Adaptation to displaced vision: A change in the central control of sensorimotor coordination. Journal of Experimental Psychology, 1971, 89, 229-239.

Harris, C. S. Perceptual adaptation to inverted, reversed, and displaced vision. Psychological Review, 1965, 72, 419-444.

HAY, J. C. Reafference learning in the presence of exafference. Perception \& Psychophysics, 1981, 30, 277-282.

HAY, J. C. Visual adaptation to an altered correlation between eye movement and head movement. Science, 1968, 160, 429430.

HELD, R. Exposure-history as a factor in maintaining stability of perception and coordination. Journal of Nervous and Mental Diseases, 1961, 132, 26-32.

HeLD, R., \& Rekosh, J. Motor-sensory feedback and the geometry of visual space. Science, 1963, 141, 722-723.

Helmholtz, H., von. Treatise on physiological optics ( J. P. C. Southall, Ed. and trans.; Vol. 3). New York: Dover, 1962. (Originally published, 1866.)
Kornheise R, A. S. Adaptation to laterally displaced vision: A review. Psychological Bulletin, 1976, 83, 783-816.

MacKay, D. M., \& Mittelstaedt, H. Visual stability and motor control (reafference revisited). In W. D. Keidel (Ed.), Cybernetics and bionics. Munich: Oldenbourg, 1974.

Martin, L., \& Müllen, G. E. Zur analyze der Untershiedsemfindlichkeit. Leipzig: Barth, 1899.

Matin, L. Eye movements and perceived visual direction. In D. Jameson \& L. Hurvich (Eds.), Handbook of sensory physiology (Vol. 7). Heidelberg: Springer-Verlag, 1972.

Matin, L. A possible hybrid mechanism for modification of visual direction associated with eye movements: The paralyzed eye experiment reconsidered. Perception, 1976, 5, 233-239.

Matin, L. Visual localization and eye movements. In W. A. Wagenaar, A. H. Wertheim, \& H. W. Leibowitz (Eds.), Symposium on the study of motion perception. New York: Plenum, 1982.

Matin, L., \& Pearce, D. G. Visual perception of direction for stimuli flashed during voluntary saccadic eye movements. Science, 1965, 148, 1485-1488.

McCloskey, D. I., Ebeling, P., \& Goodwin, G. M. Estimation of weights and tensions and apparent involvement of a "sense of effort." Experimental Neurology, 1974, 42, 220-232.

McLaughlin, S. C. Parametric adjustment in saccadic eye movements. Perception \& Psychophysics, 1967, 2, 359-362.

McLaughlin, S. C., Kelly, M. J., Anderson, R. E., \& Wenz, T. G. Localization of a peripheral target during parametric adjustment of saccadic eye movements. Perception \& Psychophysics, 1968, 4, 45-48.

Miller, J. M. Information used by the perceptual and oculomotor systems regarding the amplitude of saccadic and pursuit eye movements. Vision Research, 1980, 20, 59-68.

Miller, J. M., \& Festinger, L. Impact of oculomotor retraining on the visual perception of curvature. Journal of Experimental Psychology: Human Perception and Performance, 1977 , 3, 187-200.

Müller, G. E., \& Schuman, F. Über die psychologischen Grundlagen der Vergleichung gehobener Gewichte. Archiv fur die Gesamte Physiologie, 1889, 45, 37-112.

Ono, H., \& Nakamizo, S. Saccadic eye movements during changes in fixation to stimuli at different distances. Vision Research, 1977, 17, 233-238.

PaAP, K. R., \& Ebenholtz, S. M. Perceptual consequences of potentiation in the extraocular muscles: An alternative explanation for adaptation to wedge prisms. Journal of Experimental Psychology: Human Perception and Performance, 1976, 2, 457-468.

PaAp, K. R., \& Ebenholtz, S. M. Concomitant direction and distance aftereffects of sustained convergence: $A$ muscle potentiation explanation for eye-specific adaptation. Perception \& Psychophysics, 1977, 21, 307-314.

PolA, J. Voluntary saccades, eye position, and perceived visual direction. In R. A. Monty \& J. W. Senders (Eds.), Eye movements and psychological processes. New York: Wiley, 1976.

Rоск, I. The nature of perceptual adaptation. New York: Basic Books, 1966.

Rosinski, R. R. The development of visual perception. Santa Monica, Calif: Goodyear, 1977.

SheBILSKI, W. Visuomotor coordination in visual direction and position constancies. In W. Epstein (Ed.), Stability and constancy in visual perception. New York: Wiley, 1977. (a)

She BILSKI, W. Directional scanning biases and shifts of apparent visual direction. Vision Research, 1977, 17, 495-497. (b)

SPERry, R. W. Neural basis of the spontaneous optokinetic response produced by visual neural inversion. Journal of Comparative and Physiological Psychology, 1950, 43, 482-489.

Stevens, J. C., \& Rubin, L. L. Psychophysical scales of apparent heaviness and the size-weight illusions. Perception \& Psychophysics, 1970, 8, 225-230.

von Holst, E., \& Mittelstaedt, H. Das Reafferenzprinzip. Naturwissenshaften, 1950, 37, 464-476. 
Wallach, H., \& Kravitz, J. H. The measurement of the constancy of visual direction and of its adaptation. Psychonomic Science, 1965, 2, 217-218.

Welch, R. B. Perceptual modification. New York: Academic Press, 1978.

\section{NOTES}

1. Held's putative mechanism monitors the relationship between efference, on the one hand, and reafference, on the other, but all for the implied purpose of altering efference copies by continuously recalibrating the process by which they are generated.

2. Matin $(1972,1976,1982)$ has observed that if registered eye position is a joint function of centrifugal and centripetal impulses proceeding to and from extraocular muscle spindles, then it is an efferent/afferent hybrid. However, Matin's model could, perhaps equally well, be regarded as a pure efference model combining copies of commanded efference with copies of unrealized efference to yield a single, purely efference copy which registers centrally as realized eye position. Or, alternatively, if one entertains the possibility suggested by Goodwin et al. (1972) that corollary discharges from somatic motor areas effect sensations of movement only by altering muscle-spindle afference and are incapable of doing so on their own, perhaps Matin's model, which is fully compatible with these speculations, should be regarded as a pure afference model. It makes no difference whether one uses the terms efference/afference or input/output; the riddle remains, albeit an intriguing one. Perhaps it would be best to refer to Matin's "hybrid model" simply as Matin's model.

3. "There are almost certainly differences between the sensory processes leading to perception and those leading to saccadic eye movements"' (Hallett \& Lightstone, 1976a). For, although a stimulus flashed in darkness during a saccadic eye movement tends to be perceptually mislocalized (Matin, 1972, 1982; Matin \& Pearce, 1965), a fixation target flashed during a saccade "elicits a subsequent goal-directed saccade of normal amplitude and appropriate latency" (Hallett \& Lightstone, 1976b). Also, a saccade made during a change in fixation to a target at a different distance is directed toward the target's actual, rather than perceived, location (Ono \& Nakamizo, 1977). Apparently, some of the efference registered by the oculomotor system is not registered perceptually and is, hence, a form of unsensed efference. (However, cf. Miller, 1980.)

4. For sake of simplicity of argument, we have ignored the vertical eye movements that accompanied the subject's head nodding on the grounds that the retinal reafferences contingent upon those vertical eye movements should have corresponded to their conjugate efference copies; their net perceptual effect should have been nil.

(Manuscript received March 10, 1982; revision accepted for publication January 20,1983 .) 